\title{
Digital architecture for a piecewise-linear arbitrary-waveform generator
}

\author{
VICTOR M JIMENEZ-FERNANDEZ*, HECTOR VAZQUEZ-LEAL, PABLO S LUNA-LOZANO, \\ J L VAZQUEZ-BELTRAN, G GARCIA-SANTIAGO and E VALDES-ORTEGA
}

Facultad de Instrumentación Electrónica y Ciencias Atmosféricas, Universidad Veracruzana, Circuito Gonzalo Aguirre Beltrán s/n, Zona Universitaria, CP 91000, Xalapa, Veracruz, Mexico

e-mail: vicjimenez@uv.mx

MS received 20 March 2014; revised 16 December 2015; accepted 16 March 2016

\begin{abstract}
In this paper a digital architecture for generating piecewise-linear arbitrary waveforms is presented. The proposed design is able to generate a piecewise-linear periodic signal by only using a minimum number of input data (breakpoints). The generator circuit implements a hybrid scheme which takes advantage of two methods: the purely piecewise-linear interpolation and the lookup-table structure. From the piecewise-linear method exploits the characteristic of a reduced memory requirement as well as the capability of automatically construct a waveform by repetitive (iterative) function evaluations. From lookup-table makes use of the simplicity in hardware implementation and the higher processing speed. In order to verify the performance of this proposal, three piecewise-linear waveforms have been successfully implemented in a ATMEGA32 microcontroller. Experimental results show a fast execution speed and a reduced memory demand in the proposed circuit realization.
\end{abstract}

Keywords. Arbitrary-waveform generator; piecewise-linear; digital architecture.

\section{Introduction}

An arbitrary waveform generator (AWG) is an instrument especially suited to produce a wide variety of synthesized electrical signals. It founds application in diverse fields where a specific signal stimulus is needed to test the performance of certain devices or systems. Typical application examples of AWGs can be found in areas such as electronics, mechanics, bioengineering, physics, among others.

In recent years, several proposals for circuit synthesis of AWGs have been reported in literature being the most recurrent approach the so called direct-digital-synthesizer (DDS) which uses a lookup-table as waveform representation structure [1-9]. The DDS is based on a frequency synthesizer that operates by storing various points and then recalling them to generate the waveform. In the DDS architecture, the lookup-table is used to map the waveform in a memory whose content is cyclically addressed to generate the output waveform. Nevertheless, although this method has the capability to generate accurately arbitrary waveforms, it exhibits the disadvantage of requiring a large list of sampled waveform points to operate, what unfortunately implies a huge memory usage. To overcome this problem, an interpolation method for reconstructing a waveform from a

*For correspondence minimum set of sample points is proposed. Among all the interpolation methods, piecewise-linear is selected because of its computational advantages that make it a good option to include it in an efficient hardware design. In this paper we present a circuit realization of a programmable digital architecture with the capability of synthesizing arbitrary piecewise-linear waveforms. Our proposal is based on a linear interpolation scheme, where the circuit design exploits the advantages provided by two of the function realization approaches: piecewise-linear [10-12] and lookuptable $[13,14]$. The circuit operates in four steps: firstly, it takes as reference the minimum number of data points required to describe a piecewise-linear waveform (breakpoints), then a two's complement function is enabled to compute intermediate points between consecutive couples of breakpoints, after that, the set of evaluated points as well as the input data points are stored in memory in a lookuptable structure, and finally, a cyclical and periodical reading of the points stored in the memory table is performed.

\section{Digital piecewise-linear interpolation function}

Due to the proposed AWG circuit realization is piecewiselinear, it must be considered that all waveforms are described as a sequence of connected line segments, where 


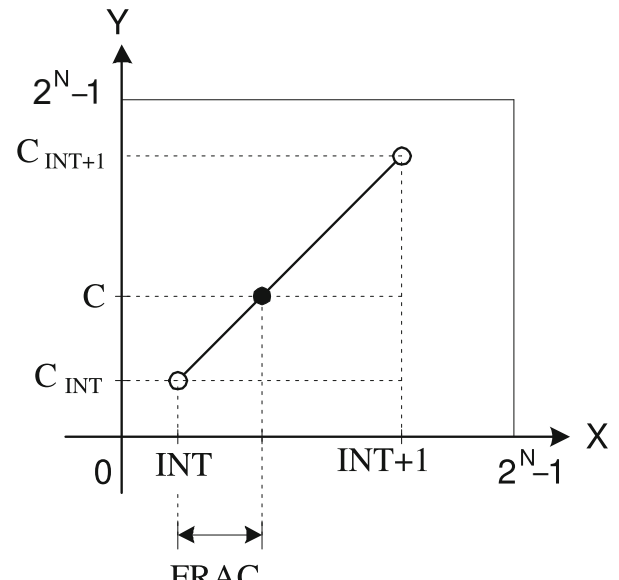

FRAC

Figure 1. Digital linear segment with N-bits precision.

each segment is defined by two data points denoted as break- points. In this sense, it can be said that the minimum information needed to construct a piecewise-linear waveform is composed by the ordered sequence of these points. Given this information, it can be observed that the problem of reproducing this kind of waveforms can also be reduced to the fact of performing a linear interpolation, starting in the leftmost breakpoint and ending to the rightmost breakpoint. The piecewise-linear interpolation is a technique that consists in finding the value of a function at unknown intermediate point given between two data points [15-17]. In order to gain insight into the geometrical interpretation for this numerical technique, the line segment depicted in figure 1 is taken as reference.

This figure represents a digital representation for a straight line with $N$-bits precision. A point, whose projection $C$ belongs to this segment, is denoted by the $2 N$-bits word INT.FRAC, where the $N$ most significant bits are used to represent the integer part (INT), and the $N$ less significant bits are used to indicate the fractional part $(F R A C)$, of the interpolated datum. Moreover, $\left(I N T, C_{I N T}\right)$ and $\left(I N T+1, C_{I N T+1}\right)$ represent the corresponding breakpoints that bound this segment.

From figure 1 the equation ratio expressed in (1) can be verified.

$$
\frac{C_{I N T+1}-C}{[I N T+1]-F R A C}=\frac{C-C_{I N T}}{F R A C-I N T} .
$$

After isolating the value $C$, it can be obtained

$$
C=\frac{C_{I N T+1}[\text { FRAC }-I N T]-C_{I N T}[\text { FRAC }-[I N T+1]]}{[I N T+1]-I N T} .
$$

Now, if we consider only integer values for the breakpoints, then the gap between INT and INT +1 will be always unitary. In this case, the value taken by $C$ will be independent of the value of INT, but it will depend exclusively on the fractional part FRAC. In this regard, a local evaluation where the conditions $I N T=0$ and $I N T+1=1$ can be considered in order to simplify Eq. (2) as follows.

$$
C=C_{I N T+1} \times F R A C-C_{I N T} \times[F R A C-1] .
$$

A factorization of (3) yields

$$
C=C_{I N T+1} \times F R A C+C_{I N T} \times[-F R A C+1] .
$$

Knowing that a two's complement is derived by taking the negative of the original number (the ones' complement) and then adding one to the result, Eq. (4) can be also expressed as

$$
C=C_{I N T+1} \times F R A C+C_{I N T} \times C M P_{2}(F R A C)
$$

where $\mathrm{CMP}_{2}(\cdot)$ is an operand to compute the two's complement.

\section{Digital architecture}

A simplified block diagram of the proposed digital architecture for the waveform generator is shown in figure 2 .

In this scheme, six main blocks can be observed:

1. CLOCK REFERENCE. The clock provides the timebase needed to synchronize the blocks. In a microcontroller implementation it can be either internal or external. In our experimental prototype, the timebase is given by an internal clock at $8 \mathrm{MHz}$.

2. RAMP GENERATOR. The ramp is a counter that increases its output up, starting in zero and ending to the $\left(2^{N}-1\right)$ value, where $N$ depends on the number of bits used to represent the fractional part (FRAC). In our case, $N=4$ what means that the ramp will run from 0 to 15 $\left(2^{4}-1=15\right)$.

3. BREAKPOINT TABLE. This is a memory bank where the breakpoints needed to describe the desired piecewise-linear waveform are stored. The size of this memory depends on the number of breakpoints used to describe the waveform (in our case 256 values per waveform).

4. ALU. This is the core element of the proposed generator scheme since it performs the logic and the arithmetic computations involved in the piecewise-linear interpolation of Eq. (5). Such operations are: two's complement $\left(C M P_{2}\right)$, multiplication (MULT) and addition $(A D D)$.

5. WAVEFORM LOOKUP TABLE. The waveform lookup table block is a memory space where all coordinate point needed to sketch the piecewise linear waveform is stored. The maximum number of available memory address will be defined in accordance with the bits resolution, in our case it is $2^{8}=256$ per waveform.

6. DAC. The function of this block consists in converting the digital data that are read from the waveform lookup- 


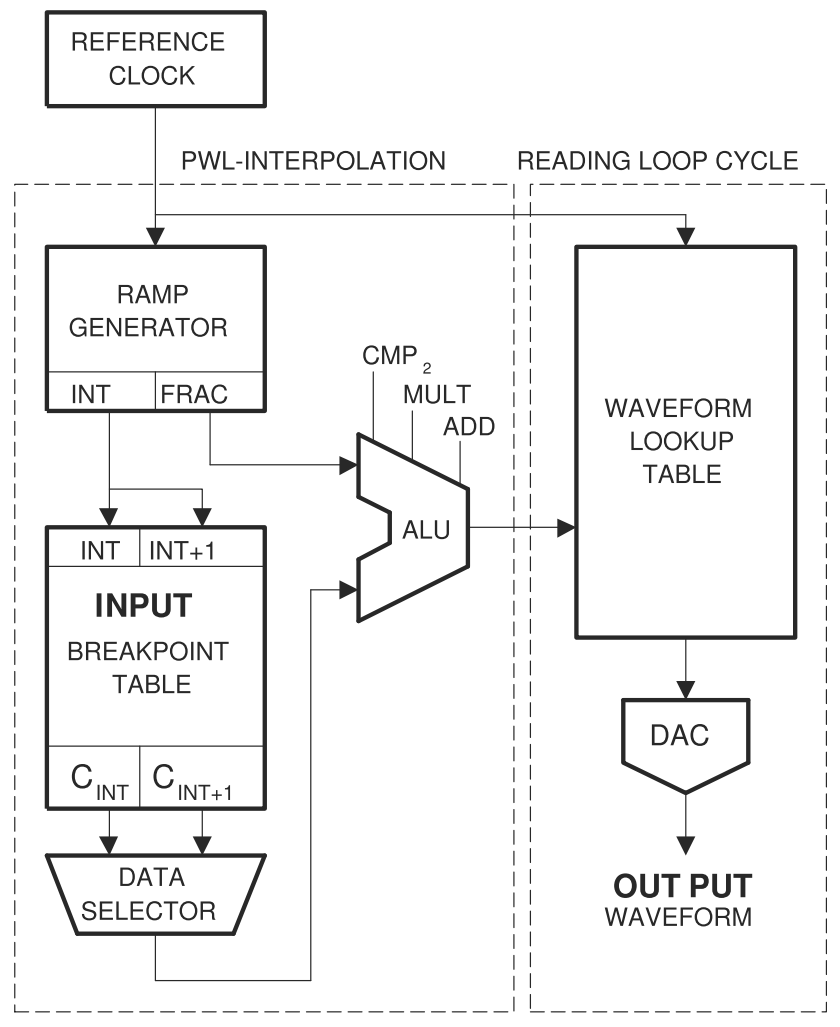

Figure 2. Digital architecture for the waveform generator.

table memory into an output analog signal. In the experimental circuit, here presented, an 8-bits DAC circuit was used.

The operation of this circuit can be summarized as follow: the waveform to be displayed is previously programmed by storing in the BREAKPOINT TABLE a minimum data set of breakpoints coordinates. The number of data values depends on two factors: the precision required in the signal sketching, and the word-length (bits precision) used to represent the integer part $(I N T)$ as well the fractional (FRAC) part, in data. In our case, a wordlength of 8-bits precision is used, the 4-bits more significant represent INT, and the 4-bits less significant represent FRAC. Once the breakpoint data is written to the BREAKPOINT TABLE memory, the RAMP GENERATOR counter counts-up clock pulses for a period of time, from 0 to $2^{N}-1$. In our scheme the count runs from 0 to 15 . The counter's output is split into two nibbles as shown in figure 2: the integer part and the fractional part. Using the integer part as address memory to access the breakpoints, the interpolant values $C_{I N T}$ and $C_{I N T}+1$ are obtained. These two points, together with the fractional part of the output counter (FRAC), are multiplexed through a data selector function with the purpose of performing $2^{\mathrm{N}}$ interpolations along to the linear segment that is bounded by the $C_{I N T}$ and $C_{I N T}+1$ values. With these operands,
Eq. (5) is recursively evaluated by the ALU block as many times as the RAMP COUNTER specifies, and each computed value is sequentially written to the WAVEFORM LOOKUP TABLE. This evaluation process is repeated until all the breakpoints have been processed. It means sweeping all the segments that compound the complete waveform. Thus far, a first stage labeled as PWL-INTERPOLATION ends to give way to the final stage so called READING LOOP CYCLE, where each data stored in the WAVEFORM LOOKUP TABLE is periodically read and sent to digital to analog converter (DAC) in order to construct the OUTPUT waveform.

\section{Implementation}

A microcontroller based prototype circuit was built to verify the waveform generator scheme of figure 2 . The hardware was mounted in a PCB which was toughly designed in order to avoid interference between tracks. The microcontroller used was the Atmel ${ }^{\circledR}$ AVR-ATmega32, programmed in C using AVR Studio v4.18.716. Practically, almost all the functional blocks of figure 2 were embedded in the microcontroller; the only external block was the DAC, directly implemented by the 8-bits digital to analog converter circuit DAC0808 series of Analog-Devices ${ }^{\mathrm{TM}}$. In fact, the internal clock of the microcontroller configured at $8 \mathrm{MHz}$, was used as reference clock. The electrical verification was done in laboratory. The power supply voltage required by the DAC circuit was provided by a 1672 -BKprecision source, and the generated waveforms were tested by a Tektronix TDS-2022-D oscilloscope as shown in figure 3 .

In order to verify the effectiveness of the proposal, the three different cases shown in figure 4, figure 5, and figure 6, were studied. Each one of these waveforms was described by a set of 16 breakpoints values. The results obtained in laboratory for the circuit prototype are presented in figure 7 , figure 8 , and figure 9 , respectively. As can be observed, a precise match between the theoretical and practical results was obtained.

\section{Results and discussion}

With the aim of estimating the performance of the proposed digital architecture, the experimental prototype is compared with two alternative hardware designs: the so-called purely PWL-Interpolation and the lookup-table. Only for comparison purposes, the proposed scheme is denoted in this section as Hybrid-PWL. For this comparative, two hardware parameters were analyzed: nominal frequency and internal memory demand. The nominal frequency is computed through the debugger tool included in the software AVR Studio. This software is able to determine the number 


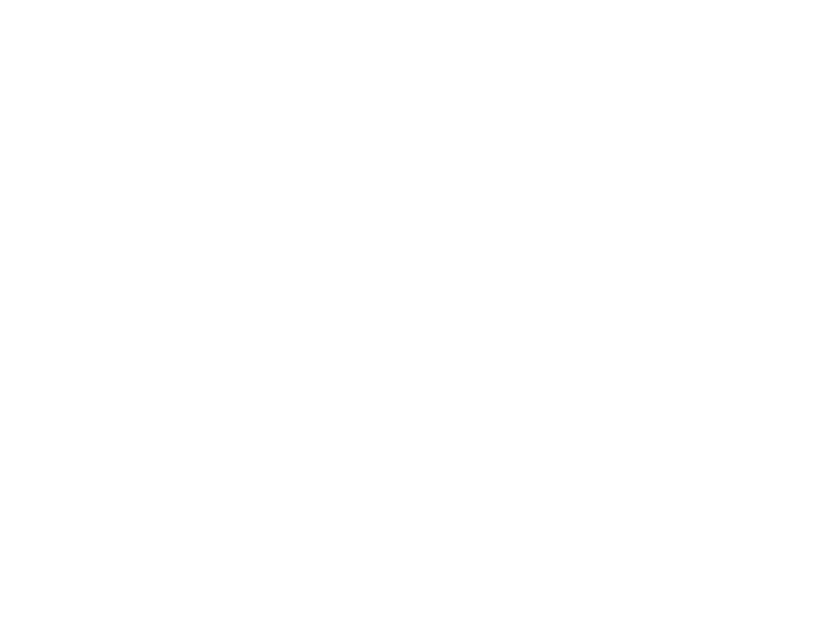

Figure 3. Electrical verification of the circuit prototype.

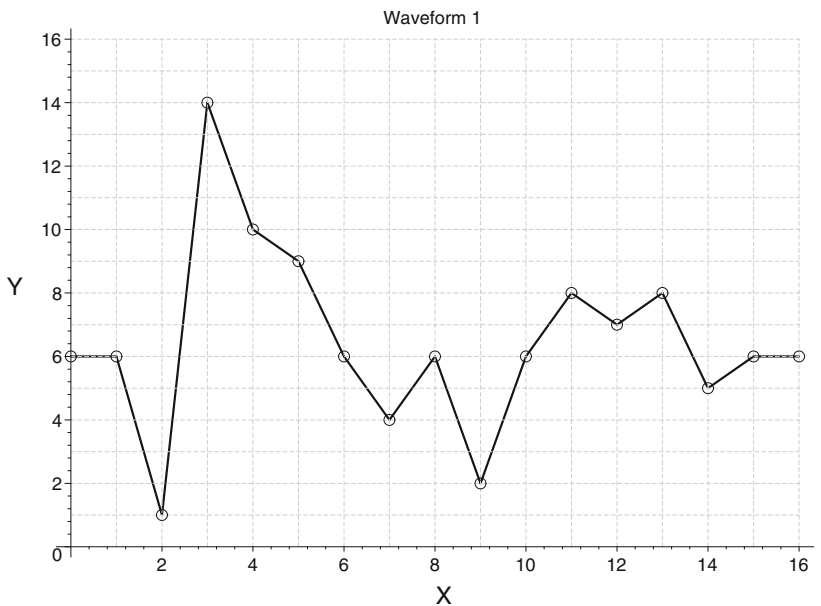

Figure 4. Waveform number 1 (WF1) for testing.

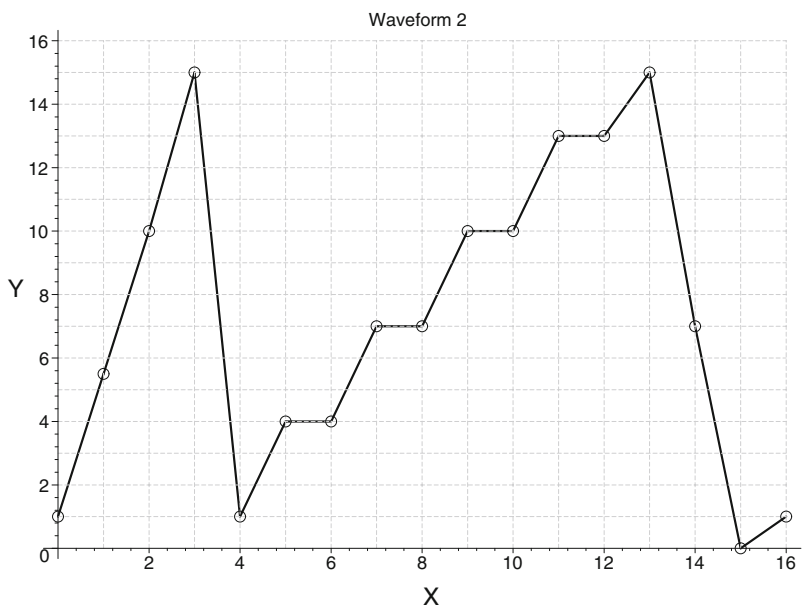

Figure 5. Waveform number 2 (WF2) for testing.

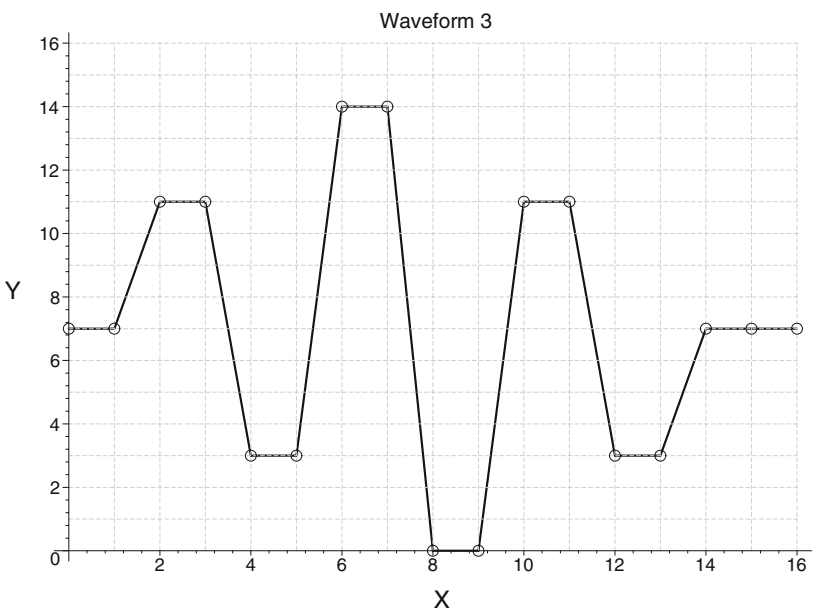

Figure 6. Waveform number 3 (WF3) for testing.

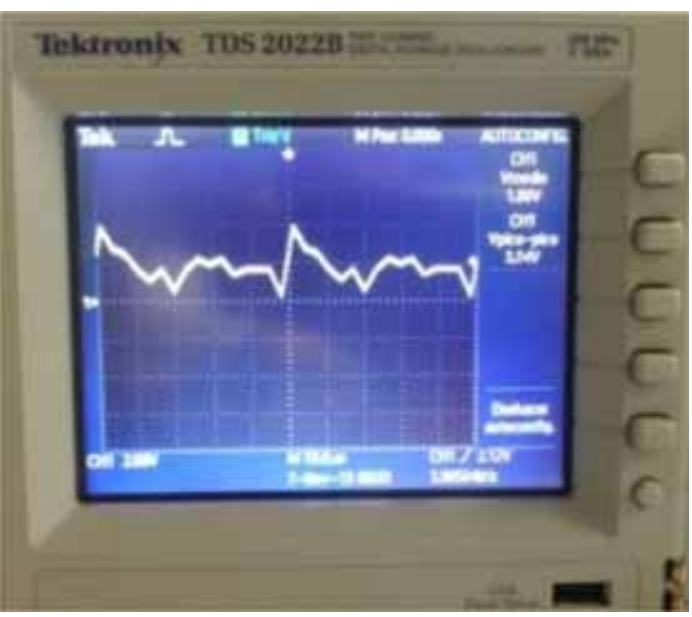

Figure 7. WF1 obtained by the circuit prototype.

of clock cycles per instruction by translating the $\mathrm{C}$ source program to assembly code in the AVR microcontroller's family. The memory demand is computed by considering the input data to program the waveform, the memory to host the program, and the output data.

\subsection{Estimation of memory demand}

The three waveforms used in this study are compound by 16 breakpoints, however, two important data must be analyzed. First, although the complete waveform is defined by 16 breakpoints, in order to ensure the line connection between the ending and the initial breakpoints, among periodic waveforms, an amount of 17 breakpoints is considered. Second, it must be clarified that this number of breakpoints was arbitrarily selected in order to have an equally distributed amount of resolution points and linear segments. That is, 17 breakpoints describe a 16 linear segments waveform, where each segment is divided into 16 


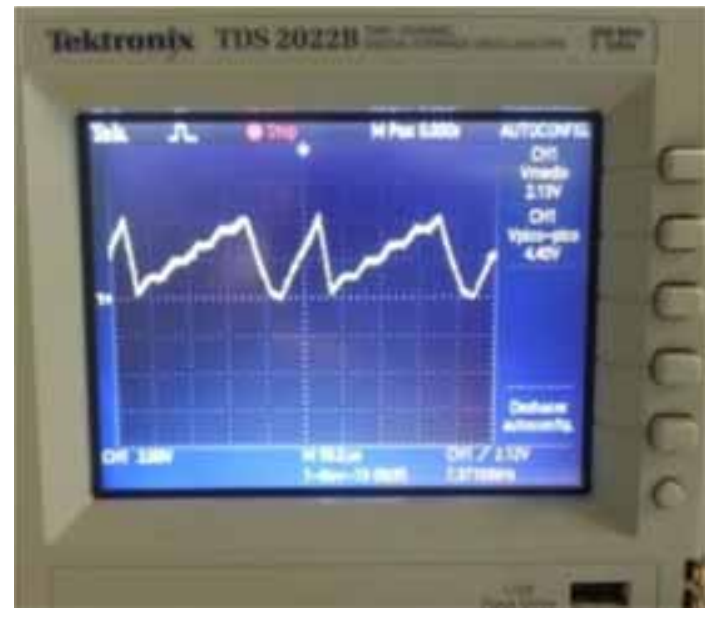

Figure 8. WF2 obtained by the circuit prototype.

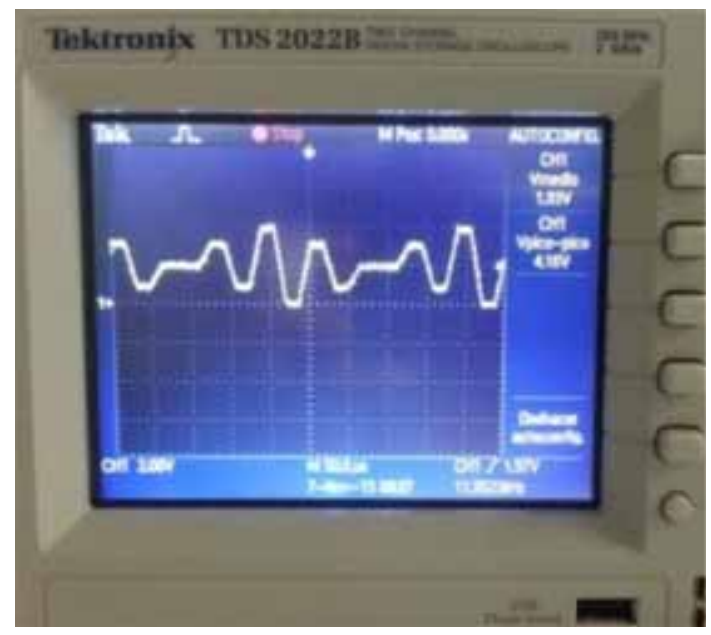

Figure 9. WF3 obtained by the circuit prototype.

interpolated values, giving as result $16 \times 16=256$ points, what is in correspondence to 8 -bits precision $\left(2^{8}=256\right)$. However, this selection can be done in a different way with the unique constraint of do not exceed the resolution of 256 points to describe a single period of the signal.

\subsection{Estimation of the nominal frequency}

In our analysis, the execution-time for the microcontroller's program is mainly considered to find the nominal frequency of the generated wave. Due to the microcontroller operates at internal clock frequency of $8 \mathrm{MHz}$, it is possible to determine the time consumed for each execution cycle as $T_{c}=1 /(8 \mathrm{MHz})=125 \mathrm{~ns}$. The proposed circuit operates in two temporal stages (shown in figure 2): a cyclic evaluation-transfer process (PWL-INTERPOLATION) and a memory reading (READING LOOP CYCLE). The evaluation requires, as input data, the breakpoints set that is
Table 1. Time and Memory comparative among the PWL-Hybrid, PWL-interpolation (PWL-I), and lookup-table approaches.

\begin{tabular}{lccccc}
\hline & \multicolumn{2}{c}{ Time } & & \multicolumn{2}{c}{ Memory } \\
\cline { 2 - 3 } \cline { 5 - 6 } Method & $T_{c}$ & $f_{r}$ & & $M_{i}$ & $N_{p}$ \\
\hline Hybrid-PWL & $254.6 \mathrm{~ms}$ & $3.9 \mathrm{KHz}$ & & 1775 bytes & 104 tables \\
PWL-I & $271.32 \mathrm{~ms}$ & $3.7 \mathrm{~Hz}$ & & 17 bytes & 120 tables \\
Lookup-table & 0 & $3.9 \mathrm{KHz}$ & & 256 bytes & 8 tables \\
\hline
\end{tabular}

stored in a microcontroller's reserved memory space. In the particular case of AVR ATMEGA32 microcontroller, a 2048-bytes memory space is reserved. The evaluationtransfer process is performed over all the breakpoints (only once time), this ensures generating all the points needed to sketch the waveform. Aided by the debugger tool it can be obtained that this process consumes 2037381 clock cycles which correspond to $254.6 \mathrm{~ms}$ for the entire waveform construction $(2037381 \times 125 \mathrm{~ns}=254.6 \mathrm{~ms})$.

The memory reading step consists in a continuous and cyclic reading process from the memory table where the interpolated data were stored. By applying the debugger tool, it can be observed that reading a single datum consumes eight clock cycles, but due to complete waveform is constituted by 256 points, therefore this reading process takes 2048 clock cycles $(256 \times 8=2048)$. Hence, it can be estimated the time of reading for a waveform as $2048 \times 125 \mathrm{~ns}=256 \mu \mathrm{s}$ where frequency is given by $f_{r}=1 /(256 \mu \mathrm{s})=3.9 \mathrm{KHz}$.

\subsection{Comparative discussion}

As previously mentioned, our proposal implements a hybrid scheme that combines two fundamental processes: a waveform construction and a cyclic reading of the drawn waveform. In order to have a comparative reference, the three waveforms taken as example are implemented by following independently each approach: on the one hand, the cyclic loop of evaluations denoted as PWL-Interpolation, and on the other hand, the cyclic memory reading strategy denoted as lookup-table. In table 1, values for reading frequency $\left(f_{r}\right)$, waveform construction time $\left(T_{c}\right)$, memory consumption $\left(M_{i}\right)$, and capability of programming additional waveforms $\left(N_{p}\right)$ are summarized.

From table 1 can be observed that the PWL-Interpolation method has the advantage of a small size memory requirement $M_{i}$ (only 17 bytes) what free up space on memory to have the capability of programming as many different tables as 120 . However, the need to continuous interpolation requires many resources of the microcontroller restricting its frequency at $3.7 \mathrm{~Hz}$. Likewise, the lookup-table method discards the task of interpolation what let it achieve a higher frequency $(3.9 \mathrm{KHz})$ but using bigger memory resources (256 bytes) what greatly limits the number of waveforms that can be programmed (only eight 
tables). In contrast, the Hybrid-PWL method intends to merge the best characteristics of both methods. As it can be seen from table 1, Hybrid-PWL reaches the same frequency as the lookup-table method, and due to the waveform construction is done only once time, it reduces the memory usage providing a programming capability as close as the PWL-Interpolation method (104 tables). Finally, it is important to note that all computations reported in table 1 were done by considering a fixed resolution of waveforms (16-segments), but it was an arbitrary selection that can be easily modified without changing the analysis results. Furthermore, the values of frequency reported in table 1 are also determined by taking as reference the internal microcontroller clock at $8 \mathrm{MHz}$ but it also can be set to other frequencies either by a frequency divider strategy or by using an oscillator as external clock.

\subsection{A comparative analysis with respect to DDS technique}

One of the standard techniques used in function arbitrary waveform generators is the so-called DDS (Direct Digital Synthesis). With DDS, one cycle of the desired waveform is represented digitally in a lookup-table memory and the contents of such memory are read sequentially to produce a stream of digital data that represents the desired waveform $[18,19]$. Although at a first glance the PWL-Interpolation proposal could be seem similar to DDS, there are two remarkable differences that must be highlighted: firstly, it must be noted that while DDS is based on a sampled strategy, PWL-Interpolation is based on a function evaluation scheme, and secondly, while with DDS a large amount of points for a single cycle of a periodic waveform must be stored in memory, with PWL-Interpolation only a minimum number of data points (breakpoints) are required to construct the desired waveform. These characteristics imply not only an important memory saving but also a lower hardware requirements. However, besides of these differences, a similar appearance could be found in both hardware structures. For example, DDS technology is implemented with three higher level hardware blocks: a sample clock, a phase accumulator, and a lookuptable [20, 21]. Similarly, PWL-Interpolation is composed of three main blocks: a reference clock, a PWL-interpolation block, and a lookup-table. In this sense, the main difference occurs between two functional blocks: the phase accumulator and the PWL-interpolations. In regards to this, it is important to clarify that while in the phase accumulator the memory address of the sample point to be output in the lookup-table is obtained from a tuning word that specifies the frequency of the signal, in its piecewise-linear counterpart the memory address is directly obtained from a simplicial evaluation procedure [22] and its operating frequency only depends on the reference clock. In accordance with literature, this characteristic let the DDS produce a wide range of periodic waveforms at many frequencies, but with the drawback of adding certain distortion to the output waveform [18]. In this respect, it can be said that in applications where a precise frequency generation is required, DDS could be an efficient solution, but in applications where the memory space is restricted and a complex user-defined waveform is needed, a better alternative solution could be PWL-Interpolation.

\section{Conclusion}

The design and implementation of an arbitrary waveform generator, specifically devoted to piecewise-linear signals, has been presented. The design is described in a digital architecture which combines and exploits the advantages of two classical methods to represent mathematical functions: the piecewise- linear interpolation and the lookup-table. The proposal has been efficiently implemented in an ATMEGA32 microcontroller system. A detailed explanation about the circuit operation as well as some experimental examples has been given. Also, it must be pointed out that due to its flexibility to generate accurately whatever piecewise-linear waveform, the circuit implementation promises applicability in a wide range of research applications. Finally, it is important to say that although the proposal, here presented, is reported for an 8-bits resolution; it can be expanded to more bits if desired without changing the fundamentals of the digital architecture design.

\section{Acknowledgment}

We gratefully acknowledge the financial support of the National Council for Science and Technology of Mexico (CONACyT) through grant CB-2010-01 no.157024.

\section{References}

[1] Adad Langlois W F and Al-Khalili D 2012 Arbitrary function generator using direct digital synthesis. Conference on Precision Electromagnetic Measurements 622-623

[2] Catunda S Y C, Saavedra O R, Fonseca Neto J V and Morais M R A 2003 Look-up table and breakpoints determination for piecewise linear approximation functions using evolutionary computation. In: Proceedings of the Twentieth IEEE Instrumentation and Measurement Technology Conference 1: 435-440

[3] Langlois J M P and Al-Khalili D 2003 Piecewise continuous linear interpolation of the sine function for direct digital frequency synthesis. IEEE MTT-S Int. Microwave Symp. Digest. 1: A65-A68

[4] Lu T L and Qiu Y L 2001 An approach to the single-chip arbitrary waveform generator (AWG). International Conference on ASIC 506-509 
[5] Ming-Gang G and Iuzzolino R J 2009 The design of direct digital frequency synthesis based on ROM lookup table. International Conference on Information Engineering and Computer Science 1-3

[6] Prasad S S 2007 Design of arbitrary waveform generator based on direct digital synthesis technique using code composer studio platform. Int. Symp. Signals Circuits Syst. 1: $1-4$

[7] Tang P T P 1991 Table-lookup algorithms for elementary functions and their error analysis. IEEE Symposium on Computer Arithmetic 232-236

[8] Weibo H, Chung-Len L and Wang X 2008 Arbitrary waveform generator based on direct digital frequency synthesizer. IEEE International Symposium on Test and Application Electronic Design 567-570

[9] Xiaodongmn L, Yanyan S and Shubo L 2007 A MCU-based arbitrary waveform generator for SLH power amplifier using DDS technique. International Conference on Electronic Measurement and Instruments 4: 895-899

[10] Ichige K, Blu T and Unser M 2006 Interpolation of signals by generalized piecewise-linear multiple generators. IEEE International Conference on Acoustics, Speech, and Signal Processing 6: 261-264

[11] Kolesnikov A 2008 Constrained piecewise linear approximation of digital curves. International Conference on Pattern Recognition 1-4.

[12] Wen C and Ma X 2008 A basis-function canonical piecewise-linear approximation. IEEE Trans. Circuits Syst. I: Regular Papers 55: 1328-1334

[13] Schulte M J and Stine J E 1997 Accurate function approximations by symmetric table lookup and addition. IEEE International Conference on Application-Specific Systems, Architectures and Processors 144-153
[14] Sobti K, Deng L, Chakrabarti C, Pitsianis N, Sun X, Kim J, Mangalagiri P, Irick K, Kandemir M and Narayanan V 2007 Efficient function evaluations with lookup tables for structured matrix operations. IEEE Workshop on Signal Processing Systems 463-468

[15] Day J and Bible S 2004 Piecewise linear interpolation on PIC12-14-16 Series Microcontrollers, Microchip, Applications Notes-AN942: 1-8

[16] Hsieh J W, Tsai G R and Lin M C 2003 Using FPGA to implement an n-channel arbitrary waveform generator with various add-on functions. IEEE International Conference on Field-Programmable Technology 296-298

[17] Rincon-Escobar T J, Bernard-Reyes C and Jimenez-Fernandez V M 2009 Arquitectura digital para la evaluacion de funciones no-lineales: una perspectiva de interpolacion triangular. In: Memorias del XXIV Congreso de Instrumentacion SOMI-2009: 43-46

[18] Agilent 2011 Comparing function generator performance: Direct digital synthesis versus point-by-point technology, URL http://cp.literature.agilent.com/litweb/pdf/5990-7460EN. pdf, online accessed on 10-December-2015

[19] Yih-Chyun J 1997 Direct digital synthesizer with jittered clock. IEEE Trans. Instrum. Measur. 46: 653-655

[20] Baochun H and Naeem S 2010 Real time DDS waveform generator in TI DSP. In: Proceedings of the fourth European DSP in education and research conference 241-244

[21] National Instruments 2015 Understanding direct digital synthesis (DDS), URL http://www.ni.com/white-paper/5516/ en/, online accessed on 11 December 2015

[22] Rodriguez J A, Lifschitz O D, Jimenez-Fernandez V M, Julian P and Agamennoni O E 2011 Application-specific processor for piecewise linear functions computation. IEEE Trans. Circuits Syst. I: 58: 971-981 